\title{
Diophantine approximation by Piatetski-Shapiro primes
}

\author{
S. I. Dimitrov
}

2020

\begin{abstract}
Let $[\cdot]$ be the floor function. In this paper we show that whenever $\eta$ is real, the constants $\lambda_{i}$ satisfy some necessary conditions, then for any fixed $1<c<38 / 37$ there exist infinitely many prime triples $p_{1}, p_{2}, p_{3}$ satisfying the inequality

$$
\left|\lambda_{1} p_{1}+\lambda_{2} p_{2}+\lambda_{3} p_{3}+\eta\right|<\left(\max p_{j}\right)^{\frac{37 c-38}{26 c}}\left(\log \max p_{j}\right)^{10}
$$

and such that $p_{i}=\left[n_{i}^{c}\right], i=1,2,3$.
\end{abstract}

Keywords: Diophantine approximation , Piatetski-Shapiro primes.

2000 Math. Subject Classification: 11D75 $\cdot 11 \mathrm{P} 32$

\section{Introduction and statement of the result}

Let $\mathbb{P}$ denotes the set of all prime numbers. In 1953 Piatetski-Shapiro [5] showed that for any fixed $\gamma \in(11 / 12,1)$ the set

$$
\mathbb{P}_{\gamma}=\left\{p \in \mathbb{P} \mid p=\left[n^{1 / \gamma}\right] \text { for some } n \in \mathbb{N}\right\}
$$

is infinite. The prime numbers of the form $p=\left[n^{1 / \gamma}\right]$ are called Piatetski-Shapiro primes of type $\gamma$. Subsequently the interval for $\gamma$ was sharpened many times and the best result up to now belongs to Rivat and Wu [6] for $\gamma \in(205 / 243,1)$.

Twenty years later Vaughan [8] proved that whenever $\delta>0, \eta$ is real and constants $\lambda_{i}$ satisfy some conditions, there are infinitely many prime triples $p_{1}, p_{2}, p_{3}$ such that

$$
\left|\lambda_{1} p_{1}+\lambda_{2} p_{2}+\lambda_{3} p_{3}+\eta\right|<\left(\max p_{j}\right)^{-\xi+\delta}
$$

for $\xi=1 / 10$. Latter the upper bound for $\xi$ was improved several times and the best result up to now is due to K. Matomäki [4] with $\xi=2 / 9$. 
In order to establish our result we solve the inequality (1) with Piatetski-Shapiro primes. Thus we prove the following theorem.

Theorem 1. Suppose that $\lambda_{1}, \lambda_{2}, \lambda_{3}$ are non-zero real numbers, not all of the same sign, $\eta$ is real, $\lambda_{1} / \lambda_{2}$ is irrational and $\gamma$ be fixed with $37 / 38<\gamma<1$. Then there exist infinitely many ordered triples of Piatetski-Shapiro primes $p_{1}, p_{2}, p_{3}$ of type $\gamma$ such that

$$
\left|\lambda_{1} p_{1}+\lambda_{2} p_{2}+\lambda_{3} p_{3}+\eta\right|<\left(\max p_{j}\right)^{\frac{37-38 \gamma}{26}}\left(\log \max p_{j}\right)^{10} .
$$

\section{Notations}

The letter $p$ will always denote prime number. As usual $[t]$ and $\{t\}$ we denote the integer part of $x$ and the fractional part of $x$. Moreover $\psi(t)=\{t\}-1 / 2$. We write $e(t)=\exp (2 \pi i t)$. Let $\gamma$ be a real constant such that $37 / 38<\gamma<1$. Since $\lambda_{1} / \lambda_{2}$ is irrational, there are infinitely many different convergents $a_{0} / q_{0}$ to its continued fraction, with

$$
\left|\frac{\lambda_{1}}{\lambda_{2}}-\frac{a_{0}}{q_{0}}\right|<\frac{1}{q_{0}^{2}}, \quad\left(a_{0}, q_{0}\right)=1, \quad a_{0} \neq 0
$$

and $q_{0}$ is arbitrary large. Denote

$$
\begin{aligned}
& X=q_{0}^{13 / 6} \\
& \Delta=X^{-\frac{12}{13}} \log X \text {; } \\
& \varepsilon=X^{\frac{37-38 \gamma}{26}} \log ^{10} X \text {; } \\
& H=\frac{\log ^{2} X}{\varepsilon} \text {; } \\
& S(\alpha, X)=\sum_{\substack{\lambda_{0} X<p \leq X \\
p \in \mathbb{P}_{\gamma}}} p^{1-\gamma} e(\alpha p) \log p, \quad 0<\lambda_{0}<1 ; \\
& \Sigma(\alpha, X)=\gamma \sum_{\lambda_{0} X<p \leq X} e(\alpha p) \log p ; \\
& \Omega(\alpha, X)=\sum_{\lambda_{0} X<p \leq X} p^{1-\gamma}\left(\psi\left(-(p+1)^{\gamma}\right)-\psi\left(-p^{\gamma}\right)\right) e(\alpha p) \log p \\
& I(\alpha, X)=\gamma \int_{\lambda_{0} X}^{X} e(\alpha y) d y
\end{aligned}
$$




\section{Preliminary lemmas}

Lemma 1. Let $\varepsilon>0$ and $k \in \mathbb{N}$. There exists a function $\theta(y)$ which is $k$ times continuously differentiable and such that

$$
\begin{array}{llc}
\theta(y)=1 & \text { for } & |y| \leq 3 \varepsilon / 4 ; \\
0<\theta(y)<1 & \text { for } & 3 \varepsilon / 4<|y|<\varepsilon ; \\
\theta(y)=0 & \text { for } & |y| \geq \varepsilon .
\end{array}
$$

and its Fourier transform

$$
\Theta(x)=\int_{-\infty}^{\infty} \theta(y) e(-x y) d y
$$

satisfies the inequality

$$
|\Theta(x)| \leq \min \left(\frac{7 \varepsilon}{4}, \frac{1}{\pi|x|}, \frac{1}{\pi|x|}\left(\frac{k}{2 \pi|x| \varepsilon / 8}\right)^{k}\right) .
$$

Proof. See (Lemma 1, [7]).

Lemma 2. Let $|\alpha| \leq \Delta$. Then for the sum denoted by (8) and the integral denoted by (10) the asymptotic formula

$$
\Sigma(\alpha, X)=I(\alpha, X)+\mathcal{O}\left(\frac{X}{e^{(\log X)^{1 / 5}}}\right)
$$

holds.

Proof. This lemma is very similar to result of Tolev [7]. Inspecting the arguments presented in ([7], Lemma 14), the reader will easily see that the proof of Lemma 2 can be obtained by the same way.

Lemma 3. For the sum denoted by (9) the upper bound

$$
\Omega(\alpha, X) \ll X^{\frac{37-12 \gamma}{26}} \log ^{5} X
$$

holds.

Proof. It follows by the same argument used in ([1], (36)). 
Lemma 4. Suppose that $\alpha \in \mathbb{R}, a \in \mathbb{Z}, q \in \mathbb{N},\left|\alpha-\frac{a}{q}\right| \leq \frac{1}{q^{2}},(a, q)=1$.

Let

$$
\Psi(X)=\sum_{p \leq X} e(\alpha p) \log p
$$

Then

$$
\Psi(X) \ll\left(X q^{-1 / 2}+X^{4 / 5}+X^{1 / 2} q^{1 / 2}\right) \log ^{4} X .
$$

Proof. See ([3], Theorem 13.6).

\section{Outline of the proof}

Consider the sum

$$
\Gamma(X)=\sum_{\substack{\lambda_{0} X<p_{1}, p_{2}, p_{3} \leq X \\ p_{i} \in \mathbb{P}_{\gamma}, i=1,2,3}} \theta\left(\lambda_{1} p_{1}+\lambda_{2} p_{2}+\lambda_{3} p_{3}+\eta\right) \log p_{1} \log p_{2} \log p_{3}
$$

Using the inverse Fourier transform for the function $\theta(x)$ we get

$$
\begin{aligned}
\Gamma(X) & =\sum_{\substack{\lambda_{0} X<p_{1}, p_{2}, p_{3} \leq X \\
p_{i} \in \mathbb{P}_{\gamma}, i=1,2,3}} \log p_{1} \log p_{2} \log p_{3} \int_{-\infty}^{\infty} \Theta(t) e\left(\left(\lambda_{1} p_{1}+\lambda_{2} p_{2}+\lambda_{3} p_{3}+\eta\right) t\right) d t \\
& =\int_{-\infty}^{\infty} \Theta(t) S\left(\lambda_{1} t, X\right) S\left(\lambda_{2} t, X\right) S\left(\lambda_{3} t, X\right) e(\eta t) d t .
\end{aligned}
$$

We decompose $\Gamma(X)$ as follows

$$
\Gamma(X)=\Gamma_{1}(X)+\Gamma_{2}(X)+\Gamma_{3}(X)
$$

where

$$
\begin{aligned}
& \Gamma_{1}(X)=\int_{|t|<\Delta} \Theta(t) S\left(\lambda_{1} t, X\right) S\left(\lambda_{2} t, X\right) S\left(\lambda_{3} t, X\right) e(\eta t) d t, \\
& \Gamma_{2}(X)=\int_{\Delta \leq|t| \leq H} \Theta(t) S\left(\lambda_{1} t, X\right) S\left(\lambda_{2} t, X\right) S\left(\lambda_{3} t, X\right) e(\eta t) d t, \\
& \Gamma_{3}(X)=\int_{|t|>H} \Theta(t) S\left(\lambda_{1} t, X\right) S\left(\lambda_{2} t, X\right) S\left(\lambda_{3} t, X\right) e(\eta t) d t .
\end{aligned}
$$

We shall estimate $\Gamma_{1}(X), \Gamma_{2}(X)$ and $\Gamma_{3}(X)$, respectively, in the sections 5, 6 and 7 , In section 8 we shall complete the proof of Theorem 1 . 


\section{Lower bound of $\Gamma_{1}(X)$}

In order to find the lower bound of $\Gamma_{1}(X)$ we need to prove the following two lemmas.

Lemma 5. For the sum denoted by (17) and the integral denoted by (10) the asymptotic formula

$$
S(\alpha, X)=I(\alpha, X)+\mathcal{O}\left(\frac{X}{e^{(\log X)^{1 / 5}}}\right)
$$

holds.

Proof. From (17) - (9) we have

$$
\begin{aligned}
S(\alpha, X) & =\sum_{\lambda_{0} X<p \leq X} p^{1-\gamma}\left(\left[-p^{\gamma}\right]-\left[-(p+1)^{\gamma}\right]\right) e(\alpha p) \log p \\
& =\sum_{\lambda_{0} X<p \leq X} p^{1-\gamma}\left((p+1)^{\gamma}-p^{\gamma}\right) e(\alpha p) \log p \\
& +\sum_{\lambda_{0} X<p \leq X} p^{1-\gamma}\left(\psi\left(-(p+1)^{\gamma}\right)-\psi\left(-p^{\gamma}\right)\right) e(\alpha p) \log p \\
& =\Sigma(\alpha, X)+\Omega(\alpha, X)+\mathcal{O}\left(\log ^{2} X\right) .
\end{aligned}
$$

Bearing in mind (17), Lemma 2 and Lemma 3 we obtain the asymptotic formula (16).

Lemma 6. Let $\lambda \neq 0$. Then for the sum denoted by (7) and the integral denoted by (10) we have

$$
\int_{-\Delta}^{\Delta}|S(\lambda \alpha, X)|^{2} d \alpha \ll X \log ^{3} X,
$$

$$
\int_{-\Delta}^{\Delta}|I(\lambda \alpha)|^{2} d \alpha \ll X \log X,
$$

Proof. We only prove (i). The inequalities (ii) and (iii) can be proved likewise. 
Using (44), (7) and Lagrange's mean value theorem we obtain

$$
\begin{aligned}
& \int_{-\Delta}^{\Delta}|S(\lambda \alpha, X)|^{2} d \alpha=\sum_{\substack{\lambda_{0} X<p_{1}, p_{2} \leq X \\
p_{i} \in \mathbb{P}_{\gamma}, i=1,2}}\left(p_{1} p_{2}\right)^{1-\gamma} \log p_{1} \log p_{2} \int_{-\Delta}^{\Delta} e\left(\lambda\left(p_{1}-p_{2}\right) \alpha\right) d \alpha \\
& \ll X^{2-2 \gamma}(\log X)^{2} \sum_{\substack{\lambda_{0} X<n_{1}, n_{2} \leq X \\
n_{i}=m_{i}^{1 / \gamma}, i=1,2}} \min \left(\Delta, \frac{1}{\left|n_{1}-n_{2}\right|}\right) \\
& \ll \Delta X^{2-\gamma} \log ^{2} X+X^{2-2 \gamma}(\log X)^{2} \sum_{\substack{\lambda_{0} X<n_{1}, n_{2} \leq X \\
n_{i}=m_{i}^{1 / \gamma}, i=1,2 \\
n_{1}<n_{2}}} \frac{1}{n_{2}-n_{1}} \\
& \ll \Delta X^{2-\gamma} \log ^{2} X+X^{2-2 \gamma}(\log X)^{2} \sum_{\substack{\left(\lambda_{0} X\right)^{\gamma}<m_{1}, m_{2} \leq X \gamma \\
m_{1}<m_{2}}} \frac{1}{m_{2}^{1 / \gamma}-m_{1}^{1 / \gamma}-1} \\
& \ll \Delta X^{2-\gamma} \log ^{2} X+X^{1-\gamma}(\log X)^{2} \sum_{\substack{\left(\lambda_{0} X\right)^{\gamma}<m_{1}, m_{2} \leq X \gamma \\
m_{1}<m_{2}}} \frac{1}{m_{2}-m_{1}} \\
& \ll \Delta X^{2-\gamma} \log ^{2} X+X \log ^{3} X . \\
& \ll X \log ^{3} X \text {. }
\end{aligned}
$$

The lemma is proved.

Put

$$
\begin{aligned}
& S_{i}=S\left(\lambda_{i} t\right), \\
& I_{i}=I\left(\lambda_{i} t\right) .
\end{aligned}
$$

We use the identity

$$
S_{1} S_{2} S_{3}=I_{1} I_{2} I_{3}+\left(S_{1}-I_{1}\right) I_{2} I_{3}+S_{1}\left(S_{2}-I_{2}\right) I_{3}+S_{1} S_{2}\left(S_{3}-I_{3}\right) .
$$

Replace

$$
J(X)=\int_{|t|<\Delta} \Theta(t) I\left(\lambda_{1} t, X\right) I\left(\lambda_{2} t, X\right) I\left(\lambda_{3} t, X\right) e(\eta t) d t
$$


Now from (13), (20), (21), Lemma 1, Lemma 5 and Lemma 6 it follows

$$
\begin{aligned}
& \Gamma_{1}(X)-J(X)=\int_{|t|<\Delta} \Theta(t)\left(S\left(\lambda_{1} t, X\right)-I\left(\lambda_{1} t, X\right)\right) I\left(\lambda_{2} t, X\right) I\left(\lambda_{3} t, X\right) e(\eta t) d t \\
& +\int_{|t|<\Delta} \Theta(t) S\left(\lambda_{1} t, X\right)\left(S\left(\lambda_{2} t, X\right)-I\left(\lambda_{2} t, X\right)\right) I\left(\lambda_{3} t, X\right) e(\eta t) d t \\
& +\int_{|t|<\Delta} \Theta(t) S\left(\lambda_{1} t, X\right) S\left(\lambda_{2} t, X\right)\left(S\left(\lambda_{3} t, X\right)-I\left(\lambda_{3} t, X\right)\right) e(\eta t) d t \\
& \ll \varepsilon \frac{X}{e^{(\log X)^{1 / 5}}}\left(\int_{|t|<\Delta}\left|I\left(\lambda_{2} t, X\right) I\left(\lambda_{3} t, X\right)\right| d t\right. \\
& \left.+\int_{|t|<\Delta}\left|S\left(\lambda_{1} t, X\right) I\left(\lambda_{3} t, X\right)\right| d t+\int_{|t|<\Delta}\left|S\left(\lambda_{1} t, X\right) S\left(\lambda_{2} t, X\right)\right| d t\right) \\
& \ll \varepsilon \frac{X}{e^{(\log X)^{1 / 5}}}\left(\int_{|t|<\Delta}\left|I\left(\lambda_{2} t, X\right)\right|^{2} d t+\int_{|t|<\Delta}\left|I\left(\lambda_{3} t, X\right)\right|^{2} d t\right. \\
& \left.+\int_{|t|<\Delta}\left|S\left(\lambda_{1} t, X\right)\right|^{2} d t+\int_{|t|<\Delta}\left|S\left(\lambda_{2} t, X\right)\right|^{2} d t\right) \\
& \ll \varepsilon \frac{X^{2}}{e^{(\log X)^{1 / 6}}} .
\end{aligned}
$$

On the other hand for the integral defined by (21) we write

$$
J(X)=B(X)+\Phi
$$

where

$$
B(X)=\gamma^{3} \int_{\lambda_{0} X}^{X} \int_{\lambda_{0} X}^{X} \int_{\lambda_{0} X}^{X} \theta\left(\lambda_{1} y_{1}+\lambda_{2} y_{2}+\lambda_{3} y_{3}+\eta\right) d y_{1} d y_{2} d y_{3}
$$

and

$$
\Phi \ll \int_{\Delta}^{\infty}|\Theta(t)|\left|I\left(\lambda_{1} t, X\right) I\left(\lambda_{2} t, X\right) I\left(\lambda_{3} t, X\right)\right| d t .
$$

According to ([2], Lemma 4) we have

$$
B(X) \gg \varepsilon X^{2}
$$


By (10) we get

$$
I(\alpha, X) \ll \frac{1}{|\alpha|} .
$$

Using (24), (26) and Lemma 1 we deduce

$$
\Phi \ll \frac{\varepsilon}{\Delta^{2}} .
$$

Bearing in mind (44), (22), (23), (25) and (27) we obtain

$$
\Gamma_{1}(X) \gg \varepsilon X^{2}
$$

\section{Upper bound of $\Gamma_{2}(\mathrm{X})$}

Suppose that

$$
\left|\alpha-\frac{a}{q}\right| \leq \frac{1}{q^{2}}, \quad(a, q)=1
$$

with

$$
q \in\left[X^{\frac{1}{13}}, X^{\frac{12}{13}}\right]
$$

Then (8), (29) and Lemma 4 yield

$$
\Sigma(\alpha, X) \ll X^{\frac{25}{26}} \log ^{4} X
$$

Now (17), (30) and Lemma 3 give us

$$
S(\alpha, X) \ll X^{\frac{37-12 \gamma}{26}} \log ^{5} X .
$$

Let

$$
\mathfrak{S}(t, X)=\min \left\{\left|S\left(\lambda_{1} t, X\right)\right|,\left|S\left(\lambda_{2} t, X\right)\right|\right\} .
$$

We shall prove the following lemma.

Lemma 7. Let $t, X, \lambda_{1}, \lambda_{2} \in \mathbb{R}$,

$$
\Delta \leq|t| \leq H
$$

where $\Delta$ and $H$ are denoted by (44) and (6), $\lambda_{1} / \lambda_{2} \in \mathbb{R} \backslash \mathbb{Q}$ and $\mathfrak{S}(t, X)$ is defined by (32). Then there exists a sequence of real numbers $X_{1}, X_{2}, \ldots \rightarrow \infty$ such that

$$
\mathfrak{S}\left(t, X_{j}\right) \ll X_{j}^{\frac{37-12 \gamma}{26}} \log ^{5} X_{j}, \quad j=1,2, \ldots
$$


Proof. Our aim is to prove that there exists a sequence $X_{1}, X_{2}, \ldots \rightarrow \infty$ such that for each $j=1,2, \ldots$ at least one of the numbers $\lambda_{1} t$ and $\lambda_{2} t$ with $t$, subject to (33) can be approximated by rational numbers with denominators, satisfying (29). Then the proof follows from (31) and (32).

Since $\lambda_{1}, \lambda_{2}, \lambda_{3}$ are not all of the same sign one can assume that $\lambda_{1}>0, \lambda_{2}>0$ and $\lambda_{3}<0$. Let us notice that there exist $a_{1}, q_{1} \in \mathbb{Z}$, such that

$$
\left|\lambda_{1} t-\frac{a_{1}}{q_{1}}\right|<\frac{1}{q_{1} q_{0}^{2}}, \quad\left(a_{1}, q_{1}\right)=1, \quad 1 \leq q_{1} \leq q_{0}^{2}, \quad a_{1} \neq 0 .
$$

From Dirichlet's approximation theorem it follows the existence of integers $a_{1}$ and $q_{1}$, satisfying the first three conditions. If $a_{1}=0$ then

$$
\left|\lambda_{1} t\right|<\frac{1}{q_{1} q_{0}^{2}}
$$

and (133) gives us

$$
\lambda_{1} \Delta<\lambda_{1}|t|<\frac{1}{q_{0}^{2}}, \quad q_{0}^{2}<\frac{1}{\lambda_{1} \Delta} .
$$

The last inequality, (3) and (41) yield

$$
X^{\frac{12}{13}}<\frac{X^{\frac{12}{13}}}{\lambda_{1} \log X}
$$

which is impossible for large $X$. Therefore $a_{1} \neq 0$. By analogy there exist $a_{2}, q_{2} \in \mathbb{Z}$, such that

$$
\left|\lambda_{2} t-\frac{a_{2}}{q_{2}}\right|<\frac{1}{q_{2} q_{0}^{2}}, \quad\left(a_{2}, q_{2}\right)=1, \quad 1 \leq q_{2} \leq q_{0}^{2}, \quad a_{2} \neq 0 .
$$

If $q_{i} \in\left[X^{\frac{1}{13}}, X^{\frac{12}{13}}\right]$ for $i=1$ or $i=2$, then the proof is completed. By (3),$(\underline{34})$ and (35) we deduce

$$
q_{i} \leq X^{\frac{12}{13}}=q_{0}^{2}, \quad i=1,2 .
$$

It remains to show that the case $q_{i}<X^{\frac{1}{13}}, i=1,2$ is impossible. Assume that

$$
q_{i}<X^{\frac{1}{13}}, \quad i=1,2 .
$$

From (15), (6)

$$
\begin{aligned}
& 1 \leq\left|a_{i}\right|<\frac{1}{q_{0}^{2}}+q_{i} \lambda_{i}|t|<\frac{1}{q_{0}^{2}}+q_{i} \lambda_{i} H, \\
& 1 \leq\left|a_{i}\right|<\frac{1}{q_{0}^{2}}+\lambda_{i} X^{\frac{38 \gamma-35}{26}}(\log X)^{-8}, \quad i=1,2 .
\end{aligned}
$$


We have

$$
\frac{\lambda_{1}}{\lambda_{2}}=\frac{\lambda_{1} t}{\lambda_{2} t}=\frac{\frac{a_{1}}{q_{1}}+\left(\lambda_{1} t-\frac{a_{1}}{q_{1}}\right)}{\frac{a_{2}}{q_{2}}+\left(\lambda_{2} t-\frac{a_{2}}{q_{2}}\right)}=\frac{a_{1} q_{2}}{a_{2} q_{1}} \cdot \frac{1+\mathfrak{X}_{1}}{1+\mathfrak{X}_{2}}
$$

where

$$
\mathfrak{X}_{i}=\frac{q_{i}}{a_{i}}\left(\lambda_{i} t-\frac{a_{i}}{q_{i}}\right), i=1,2 .
$$

Bearing in mind (34), (35), (38) and (39) we get

$$
\begin{aligned}
& \left|\mathfrak{X}_{i}\right|<\frac{q_{i}}{\left|a_{i}\right|} \cdot \frac{1}{q_{i} q_{0}^{2}}=\frac{1}{\left|a_{i}\right| q_{0}^{2}} \leq \frac{1}{q_{0}^{2}}, \quad i=1,2, \\
& \frac{\lambda_{1}}{\lambda_{2}}=\frac{a_{1} q_{2}}{a_{2} q_{1}} \cdot \frac{1+\mathcal{O}\left(\frac{1}{q_{0}^{2}}\right)}{1+\mathcal{O}\left(\frac{1}{q_{0}^{2}}\right)}=\frac{a_{1} q_{2}}{a_{2} q_{1}}\left(1+\mathcal{O}\left(\frac{1}{q_{0}^{2}}\right)\right) .
\end{aligned}
$$

Thus

$$
\frac{a_{1} q_{2}}{a_{2} q_{1}}=\mathcal{O}(1)
$$

and

$$
\frac{\lambda_{1}}{\lambda_{2}}=\frac{a_{1} q_{2}}{a_{2} q_{1}}+\mathcal{O}\left(\frac{1}{q_{0}^{2}}\right) .
$$

Therefore, both fractions $\frac{a_{0}}{q_{0}}$ and $\frac{a_{1} q_{2}}{a_{2} q_{1}}$ approximate $\frac{\lambda_{1}}{\lambda_{2}}$. Using (36), (34), (36) and inequality (37) with $i=2$ we obtain

$$
\left|a_{2}\right| q_{1}<1+\lambda_{2} X^{\frac{38 \gamma-33}{26}}(\log X)^{-8}<\frac{q_{0}}{\log X}
$$

Consequently $\left|a_{2}\right| q_{1} \neq q_{0}$ and $\frac{a_{0}}{q_{0}} \neq \frac{a_{1} q_{2}}{a_{2} q_{1}}$. Now (41) implies

$$
\left|\frac{a_{0}}{q_{0}}-\frac{a_{1} q_{2}}{a_{2} q_{1}}\right|=\frac{\left|a_{0} a_{2} q_{1}-a_{1} q_{2} q_{0}\right|}{\left|a_{2}\right| q_{1} q_{0}} \geq \frac{1}{\left|a_{2}\right| q_{1} q_{0}}>\frac{\log X}{q_{0}^{2}} .
$$

On the other hand, from (2) and (40) we deduce

$$
\left|\frac{a_{0}}{q_{0}}-\frac{a_{1} q_{2}}{a_{2} q_{1}}\right| \leq\left|\frac{a_{0}}{q_{0}}-\frac{\lambda_{1}}{\lambda_{2}}\right|+\left|\frac{\lambda_{1}}{\lambda_{2}}-\frac{a_{1} q_{2}}{a_{2} q_{1}}\right| \ll \frac{1}{q_{0}^{2}},
$$

which contradicts (42). This rejects the assumption (36). Let $q_{0}^{(1)}, q_{0}^{(2)}, \ldots$ be an infinite sequence of values of $q_{0}$, satisfying (2). Then using (3) one gets an infinite sequence $X_{1}, X_{2}, \ldots$ of values of $X$, such that at least one of the numbers $\lambda_{1} t$ and $\lambda_{2} t$ can be approximated by rational numbers with denominators, satisfying (29). Hence, the proof is completed. 
Taking into account (14), (32), Lemma 1 and Lemma 7 we deduce

$$
\begin{aligned}
\Gamma_{2}\left(X_{j}\right) & \ll \varepsilon \int_{\Delta \leq|t| \leq H} \mathfrak{S}\left(t, X_{j}\right)\left(\left|S\left(\lambda_{1} t, X_{j}\right) S\left(\lambda_{3} t, X_{j}\right)\right|+\left|S\left(\lambda_{2} t, X_{j}\right) S\left(\lambda_{3} t, X_{j}\right)\right|\right) d t \\
& \ll \varepsilon \int_{\Delta \leq|t| \leq H} \mathfrak{S}\left(t, X_{j}\right)\left(\left|S\left(\lambda_{1} t, X_{j}\right)\right|^{2}+\left|S\left(\lambda_{2} t, X_{j}\right)\right|^{2}+\left|S\left(\lambda_{3} t, X_{j}\right)\right|^{2}\right) d t \\
& \ll \varepsilon X_{j}^{\frac{37-12 \gamma}{26}}\left(\log X_{j}\right)^{5} T_{k},
\end{aligned}
$$

where

$$
T_{k}=\int_{\Delta}^{H}\left|S\left(\lambda_{k} t, X_{j}\right)\right|^{2} d t .
$$

Using Lemma 6 (iii) and working as in ([2], p. 17-18) we obtain

$$
T_{k} \ll H X_{j}^{2-\gamma} \log ^{2} X_{j}
$$

From (5), (6), (43), (44) we get

$$
\Gamma_{2}\left(X_{j}\right) \ll X_{j}^{\frac{37-12 \gamma}{26}} X_{j}^{2-\gamma} \log ^{9} X_{j} \ll X_{j}^{\frac{89-38 \gamma}{26}} \log ^{9} X_{j} \ll \frac{\varepsilon X_{j}^{2}}{\log X_{j}} .
$$

\section{$7 \quad$ Upper bound of $\Gamma_{3}(X)$}

By (7), (15) and Lemma 1 it follows

$$
\Gamma_{3}(X) \ll X^{3-3 \gamma} \int_{H}^{\infty} \frac{1}{t}\left(\frac{k}{2 \pi t \varepsilon / 8}\right)^{k} d t=\frac{X^{3-3 \gamma}}{k}\left(\frac{4 k}{\pi \varepsilon H}\right)^{k} .
$$

Choosing $k=[\log X]$ from (6) and (46) we obtain

$$
\Gamma_{3}(X) \ll 1 \text {. }
$$

\section{Proof of the Theorem}

Summarizing (5), (12), (28), (45) and (47) we deduce

$$
\Gamma\left(X_{j}\right) \gg \varepsilon X_{j}^{2}=X_{j}^{\frac{89-38 \gamma}{26}} \log ^{10} X_{j} .
$$

The last estimation implies

$$
\Gamma\left(X_{j}\right) \rightarrow \infty \quad \text { as } \quad X_{j} \rightarrow \infty .
$$

Bearing in mind (11) and (48) we establish Theorem 1. 


\section{References}

[1] S. I. Dimitrov, On the distribution of ap modulo one over Piatetski-Shapiro primes, arXiv:2005.05008v1 [math.NT] 11 May 2020.

[2] S. Dimitrov, T. Todorova, Diophantine approximation by prime numbers of a special form, Annuaire Univ. Sofia, Fac. Math. Inform., 102, (2015), 71 - 90.

[3] H. Iwaniec, E. Kowalski, Analytic number theory, Colloquium Publications, 53, Amer. Math. Soc., (2004).

[4] K. Matomäki, Diophantine approximation by primes, Glasgow Math. J., 52, (2010), $87-106$.

[5] I. I. Piatetski-Shapiro, On the distribution of prime numbers in sequences of the form $[f(n)]$, Mat. Sb., 33, (1953), $559-566$.

[6] J. Rivat, J. Wu, Prime numbers of the form [ $\left.n^{c}\right]$, Glasg. Math. J, 43, 2, (2001), 237 254.

[7] D. Tolev, On a diophantine inequality involving prime numbers, Acta Arith., 61, (1992), $289-306$.

[8] R. Vaughan, Diophantine approximation by prime numbers I, Proc. Lond. Math.Soc., 28(3), (1974), $373-384$.

S. I. Dimitrov

Faculty of Applied Mathematics and Informatics

Technical University of Sofia

8, St.Kliment Ohridski Blvd.

1756 Sofia, BULGARIA

e-mail: sdimitrov@tu-sofia.bg 